\title{
UHF RFID Antenna: Printed Dipole Antenna with a CPS Matching Circuit and Inductively Coupled Feed
}

\author{
Regular Paper
}

\author{
Nenad Popović \\ 1 IMTEL Institute, Belgrade, Serbia \\ Corresponding author E-mail: nenad@insimtel.com
}

Received 9 May 2011; Accepted 22 June 2011

\begin{abstract}
This paper presents simulated (WIPL-D pro) and measured results of a UHF RFID antenna realized with a dipole matched to a CPS (Coplanar Stripline) and inductively coupled with a small rectangular loop. Such a design enables achieving and controlling high values of the inductive reactance that is necessary for obtaining good match of the antenna to an Application Specific Integrated Circuit (ASIC) chip. The antenna is characterized by a simple and robust design, which results in low-cost realization.
\end{abstract}

Keywords Radio Frequency Identification, Antennas, Printed Dipole.

\section{Introduction}

Radio Frequency Identification (RFID) is an automatic wireless technology for data transfer developed in the 1970s. This technology has been developing in recent years and has become popular in many service industries, purchasing and distribution logistics, industry, manufacturing companies and material flow systems. It also provides information about people, animals, goods and products in transit etc. There are several standards that regulate the use of RFID systems depending on region (ISO, Class0, Class1 i Gen 2) [1].
RFID systems consist of two components: the reader and the transponder (or tag). There are two main classes of RFID systems, depending on characteristics of the transponder: active and passive. Passive RFID systems, which are more frequent, use the electromagnetic field of the reader for their operation, because the passive transponders do not have their own power supply. Active transponders incorporate a battery which supplies all or part of the power for the operation.

In digital data transmission between the reader and the transponder, in a full duplex system, the following three types of modulation are mostly used:

- ASK (Ampitude Shift Keying),

- FSK (Frequency Shift Keying),

- $\quad$ PSK (Phase Shift Keying).

Due to its simple demodulation, ASK is commonly used. The features of any wireless system, including RFID, depend highly on the characteristics of the antenna (a) and the propagation channel (b):

(a) Operating frequency, gain characteristics (maximum gain, radiation pattern, beamwidth, etc.), matching (VSWR or return loss), polarization, sensitivity to nearby objects with different properties. 
(b) Path loss, fading.

Depending on the wireless communication system, some of these characteristics may be more important factors in design of RFID systems. Thus, operation of passive UHF RFID systems is based on modulation of the reflected RF wave or backscatter from the reader, that differs from modulations in traditional wireless systems, which comprise active transcievers on both sides of the link (802.11, Bloutooth, etc).

A passive transponder consists of two main parts: the antenna, and the application specific integrated circuit (ASIC). All the energy needed for the operation of the microchip has to be provided from the RF signal received from the antenna. The information necessary for the identification of the object carrying the transponder is written in the ASIC chip. This digital information with precisely defined content and length is stored in an appropriate RAM and written in the ASIC chip memory.

Hence, the modulated RF signal emitted from the reader's antenna reaches the transponder's antenna. Part of this energy is used for the transponder's operation by changing its input impedance, according to the modulation information written in ASIC chip. In the return link from transponder to the reader, the incoming $\mathrm{RF}$ signal is backscattered from the transponder and a proportion of incoming power is returned to the reader's antenna. The processing circuit of the transponder (ASIC) alters its RF impedance and controls the amount of the reflected field. In this case, modulation of the scattered field contains the identification information. The transponder is identified when the reflected field is received and decoded in the reader's unit. The antennas play a crucial role in such communication system; thus, great attention has to be paid to their design. Some of the main transponder antenna characteristics include:

- They must have dimensions small enough to be attached to the required object,

- They must have omnidirectional or hemispherical radiation pattern,

- They must provide a maximum possible signal to the ASIC,

- $\quad$ They must have a polarization that matches the enquiry signal regardless of the physical orientation of the object,

- They must be robust,

- $\quad$ They must be inexpensive.

Due to their simple design, small dimensions and good electrical characteristics, printed dipole antennas are frequently used in realization of passive RFID (radiofrequency identification) transponders (or tags) operating in UHF (866-956 MHz) (Table 1.) and microwave (2.4 $\mathrm{GHz}$ and $5.8 \mathrm{GHz}$ ) ranges [1]. Omnidirectional radiation pattern of a dipole is suitable because it enables reliable object identification regardless of its position in the observed space.

\begin{tabular}{|l|l|l|l|}
\hline Region & $\begin{array}{l}\text { Frequency } \\
\text { Range }\end{array}$ & Wavelength & Bandwidth \\
\hline Europe & $866-869 \mathrm{MHz}$ & $0.346-0345 \mathrm{~m}$ & $3 \mathrm{MHz}$ \\
America & $902-928 \mathrm{MHz}$ & $0.332-0.323 \mathrm{~m}$ & $26 \mathrm{MHz}$ \\
Asia & $950-956 \mathrm{MHz}$ & $0.315-0.318 \mathrm{~m}$ & $6 \mathrm{MHz}$ \\
\hline
\end{tabular}

Table 1. UHF RFID frequency ranges for different regions.

Matching of the antenna input impedance to an ASIC (Application Specific Integrated Circuit) chip (that form together a transponder) is of special importance because the reliability and range of identification depends directly on the quality of matching.

Since input impedance of ASIC chips is usually characterized by considerable capacitive reactance (most often in the range from $-100 \Omega$ to about $-400 \Omega$ ), and a real part of the impedance which is one order of magnitude smaller than its imaginary part (Table 2.), input reactance of the antenna has to be inductive and of the same order of magnitude as the reactance of the used microchip (i.e. their impedances have to be complex-conjugated), in order to achieve good matching between them [2].

\begin{tabular}{|l|l|l|}
\hline Chip & Impedance $(\Omega)$ & Manufacturers \\
\hline MM9647 & $73-j 113 / 915 \mathrm{MHz}$ & NSC \\
ALL-9238 & $20-\mathrm{j} 127 / 900 \mathrm{MHz}$ & Alien Technology \\
ALN-9338-R & $6.2-\mathrm{j} 127 / 915 \mathrm{MHz}$ & Alien Technology \\
EPC1.19G2 & $16-\mathrm{j} 315 / 914 \mathrm{MHz}$ & Philips \\
\hline
\end{tabular}

Table 2. Input impedance of various ASIC chips.

Dipole with a Coplanar Strip (CPS) matching [3] [4] [5] [6] is characterized by an inductive input reactance that makes it very suitable for application in passive UHF RFID transponders.

As a first step we have analyzed the dipole with a CPS matching and investigated dependence of input impedance with variation of its characteristic physical dimensions, primarily of dipole's arm length and a CPS line length. The analysis has been carried out using WIPL-D pro software package [7].

In the next step, we have examined the influence of a rectangular loop, inductively coupled with the dipole, on input impedance of the antenna structure formed in such manner. Dimensions of the loop and strength of inductive coupling have been varied and effect of these variations on the antenna input impedance has been analyzed.

Finally, the method for measuring the antenna input impedance, that was carried out using Agilent Technologies E8364A network analyzer, is presented. 


\section{Printed Half-wavelength Dipole with a CPS Matching}

The structure of the printed half-wavelength dipole $(\mathrm{L} \cong$ $\lambda / 2$ ) with a feed line which enters into the dipole arm forming a CPS line is presented in Fig. 1. [3]. In such manner (by modifying the depth of the feed line penetration in the dipole arm) it is possible to change the input impedance of the dipole, beside usual ways of its variation - by changing the dipole arm's width and length.

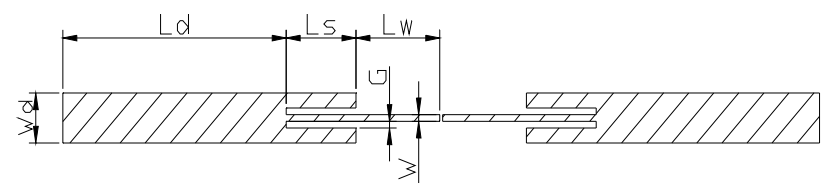

Figure 1. Sketch of the dipole.

This type of a half-wavelength dipole is characterized by predominantly inductive input reactance when its length is less than $\lambda / 2$, differing from a regular printed dipole having the capacitive reactance. This feature of a modified dipole makes it very convenient for applications in UHF RFID transponders when its input impedance has to be matched to the input impedance of a transponder's chip that usually has significant capacitive reactance.

The overall length of the dipole's arm L (Fig. 1.) is a sum of lengths Ld, Ls and Lw that contribute together in the antenna radiation. Figure 2. shows the simplified equivalent circuit of the antenna.

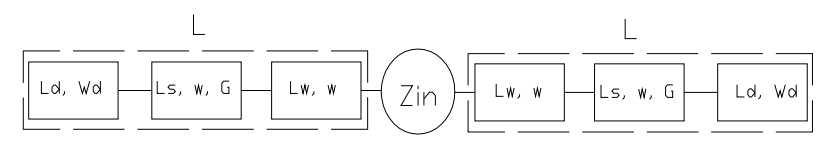

Figure 2. Simplified equivalent circuit of the antenna.

Important characteristic of an antenna is its input impedance Zin. The input impedance of this type of antenna is a function of the following dipole's dimensions: Ld, Ls, Lw, Wd, G and w. Each of them has its specific influence on the input impedance variation, however some of them being more influenceable.

The following diagrams (Fig. $3(a, b, c, d, e)$ ) present the effect of variation of each of dipole's dimensions on the input impedance (Zin). Diagrams are plotted for the central frequency $(900 \mathrm{MHz})$ of the UHF RFID range (850950) $\mathrm{MHz}$.

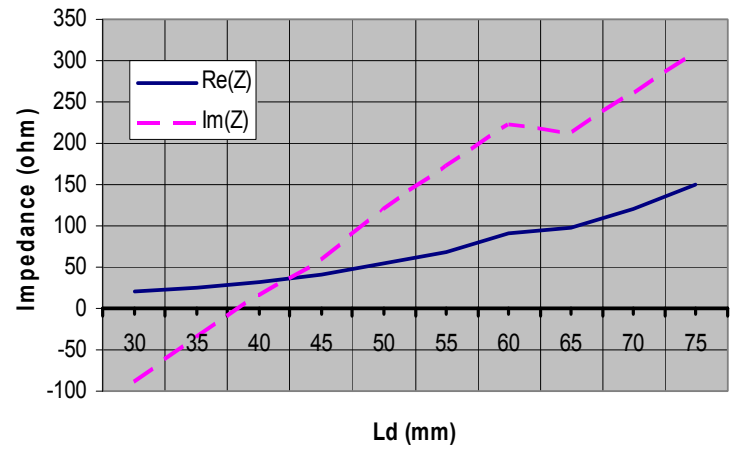

Figure 3. (a) Diagrams of the antenna input impedance variation vs. dipole's dimension Ld.

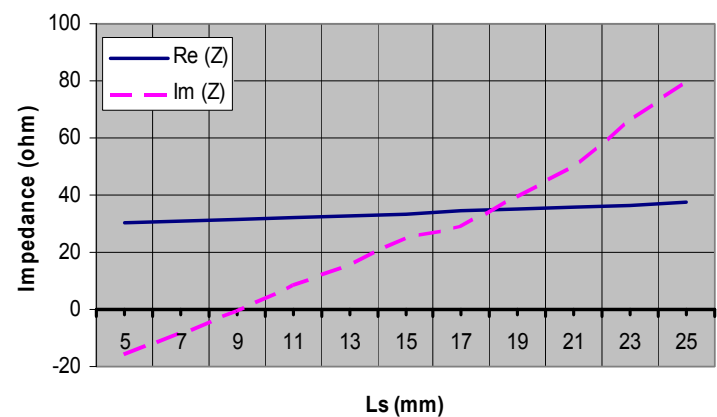

Figure 3. (b) Diagrams of the antenna input impedance variation vs. dipole's dimension Ls.

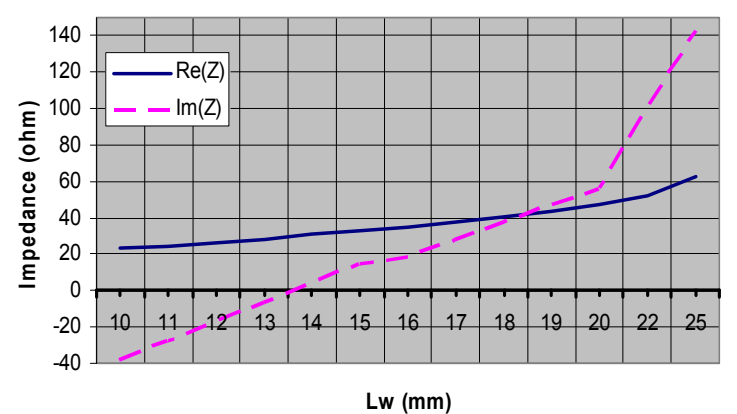

Figure 3. (c) Diagrams of the antenna input impedance variation vs. dipole's dimension Lw.

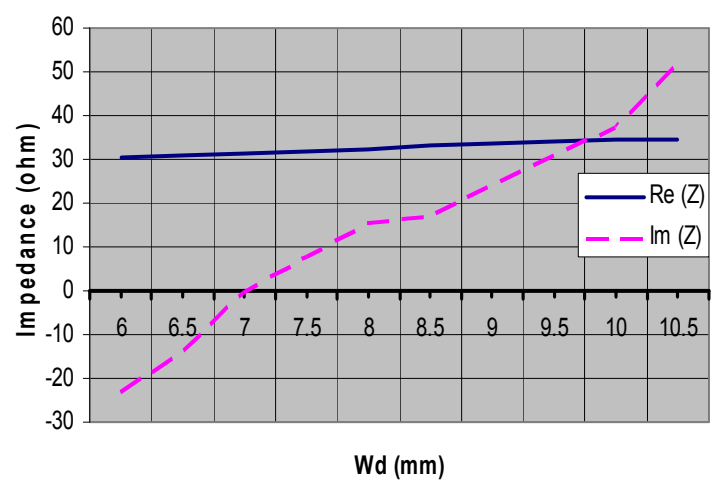

Figure 3. (d) Diagrams of the antenna input impedance variation vs. dipole's dimension $\mathrm{Wd}$. 


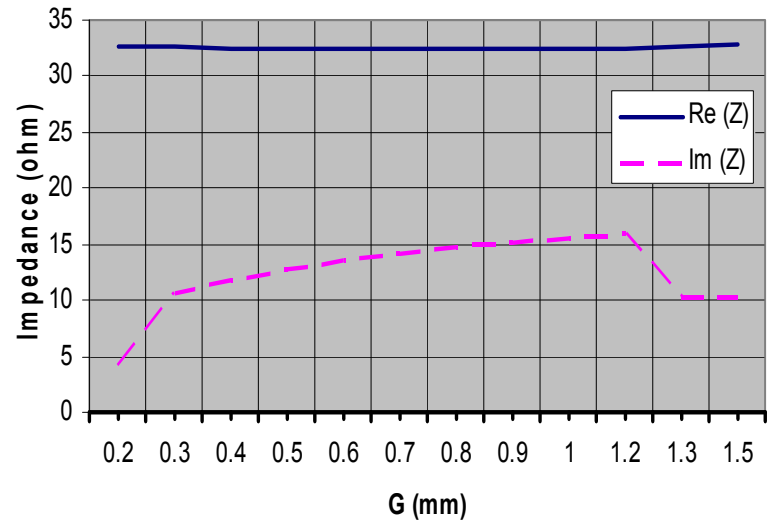

Figure 3 (e). Diagrams of the antenna input impedance variation vs. dipole's dimension $\mathrm{G}$.

The dipole is realized on the dielectric substrate with a dielectric constant $\varepsilon_{\mathrm{r}}=3.38$ and thickness of $0.2 \mathrm{~mm}$ and is printed on one side of the substrate.

The analysis [7] has been carried out by varying one of the dimensions at a time while keeping the others unchanged $(\mathrm{Ld}=40 \mathrm{~mm}, \mathrm{Ls}=12.5 \mathrm{~mm}, \mathrm{Lw}=15 \mathrm{~mm}, \mathrm{Wd}=8$ $\mathrm{mm}, \mathrm{G}=1 \mathrm{~mm}$ and $\mathrm{w}=1 \mathrm{~mm}$ ). From the diagrams in Fig. 3 . (especially observing the reactance variation) it is obvious that change of the gap $(G)$ dimension has the least influence, while changing Ld, Lw and Ls produces remarkable effect on the input impedance reactance. On the other hand, variation of the length $\mathrm{Ld}$ causes significant change of the real part of the input impedance. With suitable combination of these dimensions it is possible to achieve very wide range of impedance values and in this way find the one that provides the best matching with transponder's chip impedance.

\section{Printed Dipol Antenna With a CPS Matching Circuit and Inductively Coupled Feed}

\subsection{Transponder Antenna Design}

Two characteristics of transponder antenna are especially important: the input impedance and the radiation pattern. The input impedance of the antenna has to be matched to the chip impedance; in other words, these two impedances have to be complex-conjugates. Most of chips have the real part of their impedance approximately a few to several dozens of ohms and a capacitive imaginary part of approximately 100 to $1000 \Omega$. The antenna must have an impedance with a small real part and with an inductive imaginary part of relatively high value. It should be noted that it is very important to provide a good match between the antenna and the ASIC chip, in order to achieve, as efficient as possible, the RF energy transmission from the antenna to the chip. The chip in passive RFID systems also uses this energy for its power supply.
The radiation pattern, as already mentioned, has to be omnidirectional. A dipole antenna fulfills this requirement relatively easily; it is usually used in various versions of RFID transponders. Dipoles have a simple structure, so antennas realized with them are not complex and therefore are convenient for in-series production.

For the realization of the antenna, a dipole with CPS matching has been chosen [6], which enables high values of inductive reactance. By changing its geometry, that is the length of the dipole arm (Ld) and the length of the matching CPS (Ls), it is easy to control both the real and imaginary part of the dipole's input impedance. In order to achieve greater values of the inductive reactance, one more element - an inductively coupled rectangular loop was also introduced that enables additional varying and controlling of the antenna's input impedance. Fig. 4 shows the layout of the designed antenna. By optimizing the loop's length (Lp) and its distance from the feed line (h), the required value of the input impedance can be easily obtained.

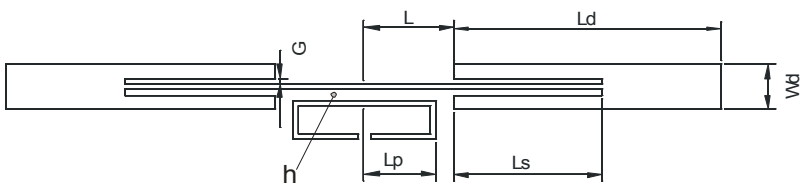

Figure 4. Layout of the designed antenna with a CPS matching and inductively coupled loop.

\subsection{Realization of the RFID Antenna}

The RFID antenna is designed for the ASIC chip input impedance of $Z_{c}=(20-j 127) \Omega$ at $900 \mathrm{MHz}$ (ALL-9238 from Alien Technology) and a dielectric substrate (Rogers RO4003C) $(\varepsilon r=3.38, h=0.2 \mathrm{~mm})$. First, only the dipole was analyzed by varying its dimensions, using the program package for electromagnetic simulation. After satisfactory results (high values of inductive reactance and low values of resistance), were obtained, the complete antenna structure, together with an inductively coupled loop, was analyzed. The input antenna impedance attained by simulation is approximately $(18+\mathrm{j} 124) \Omega$ at $900 \mathrm{MHz}$ for the following antenna dimensions: $\mathrm{Ld}=45 \mathrm{~mm}, \mathrm{Wd}=8$ $\mathrm{mm}, \mathrm{L}=15 \mathrm{~mm}, \mathrm{Ls}=25 \mathrm{~mm}, \mathrm{~h}=1.5 \mathrm{~mm}, \mathrm{Lp}=12 \mathrm{~mm}$. The width of the feed line is $1 \mathrm{~mm}$. Fig. 5 shows the realized antenna with ASIC chip.

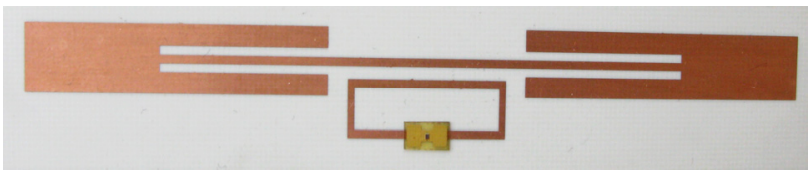

Figure 5. Realized antenna with ASIC chip. 


\subsection{Simulated and Measured Results}

The analysis consisted of varying one of the characteristic dimensions at a time, while keeping the others constant, in order to obtain the required impedance value. The diagrams in Fig. 6 show the results of the analysis using [7].

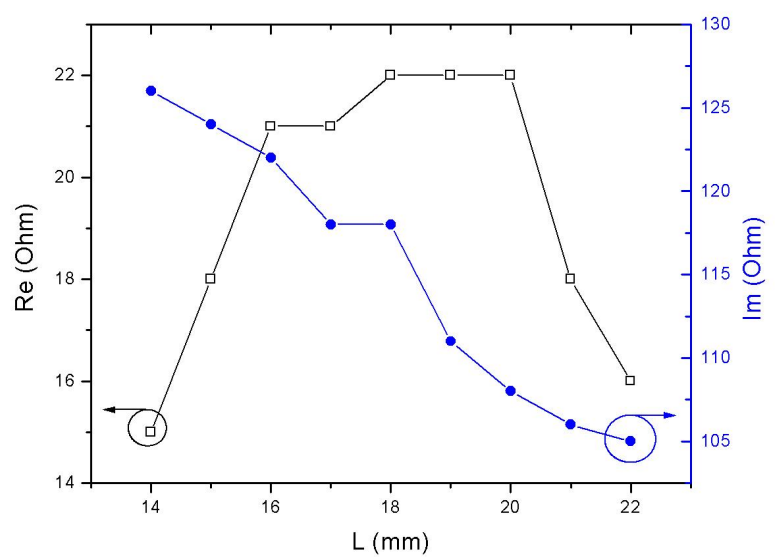

Figure 6. (a) Results of the analysis of influence of the antenna dimension $L$ on its input impedance.

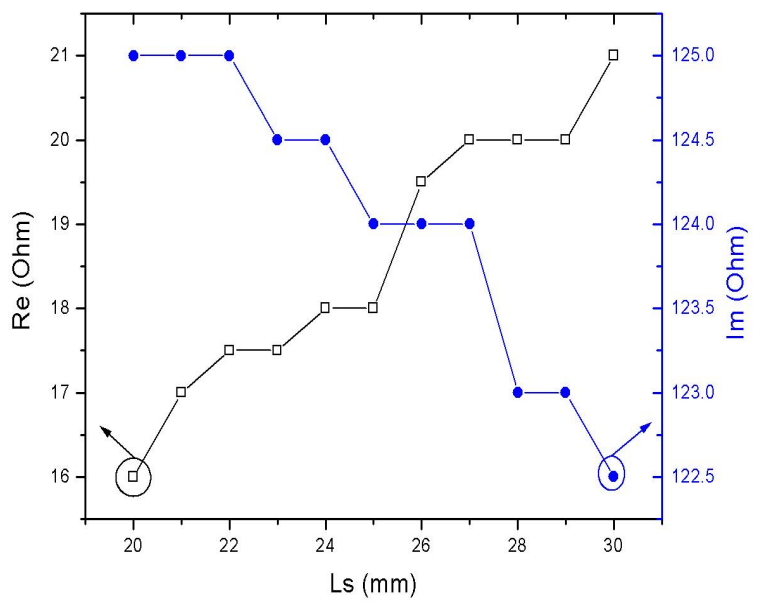

Figure 6. (b) Results of the analysis of influence of the antenna dimension Ls on its input impedance.

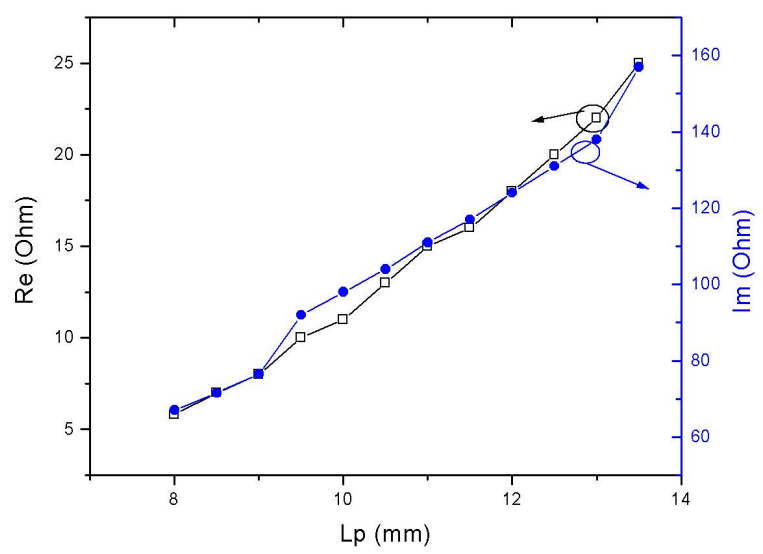

Figure 6. (c) Results of the analysis of influence of the antenna dimension Lp on its input impedance.
From the accomplished analysis, one can conclude that the rectangular loop's length (Lp) as well as the distance from the feed line $(\mathrm{h})$ have the most significant effect on the impedance variation. The simulated radiation patterns in E-plane and H-plane are presented in Fig. 7. $(a, b)$.

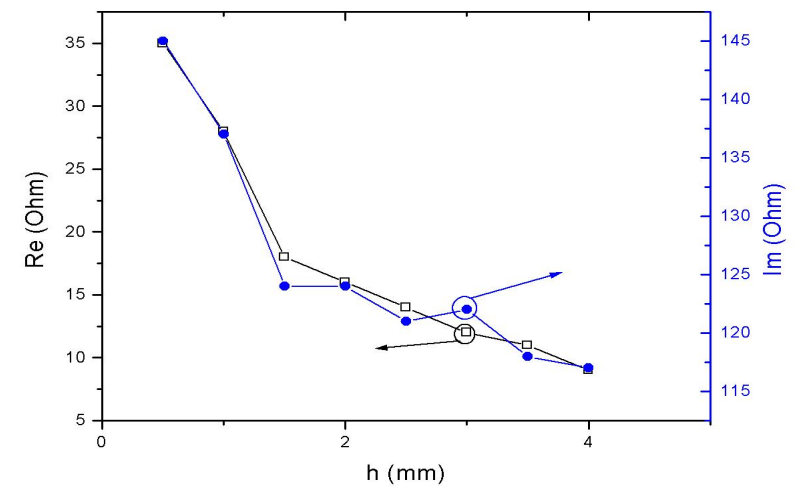

Figure 6. (d) Results of the analysis of influence of the loop distance $\mathrm{h}$ from the feed line on the antenna input impedance.

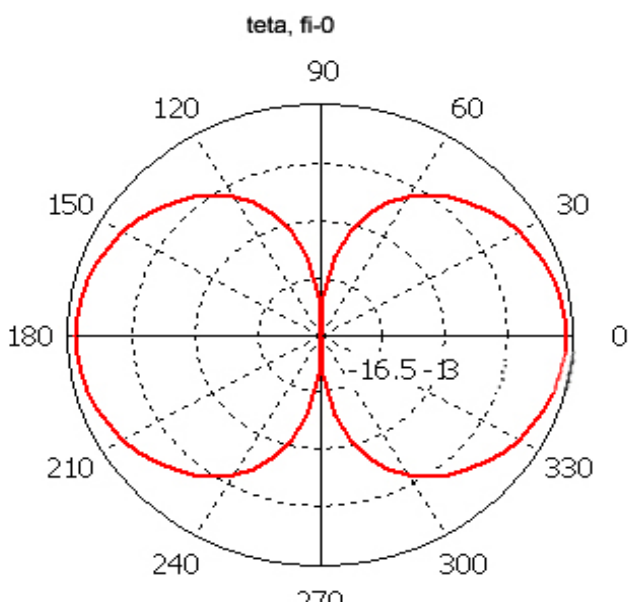

Figure 7. (a) Simulated H-plane radiation patterns of the UHF RFID antenna with inductively coupled loop.

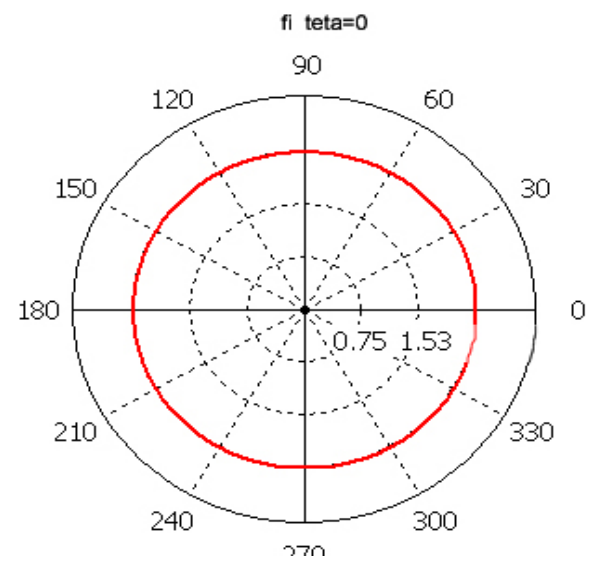

Figure 7. (b) Simulated E-plane radiation patterns of the UHF RFID antenna with inductively coupled loop. 
The simulated gain of the antenna is $2.45 \mathrm{dBi}$.

Due to the symmetrical input port and the significantly high inductive reactance, the particular problem concerning this antenna type is the input impedance measurement. One solution is to transform the symmetrical input to an unsymmetrical one [8]; the other is to use just one half of the antenna placed above conducting grounded plane and thus obtain an unsymmetrical input [9]. The latter method is simpler and does not introduce additional losses. In this way, the measured impedance is equal to one half of the whole antenna impedance. The method of measuring one half of the antenna is shown in Fig. 8.

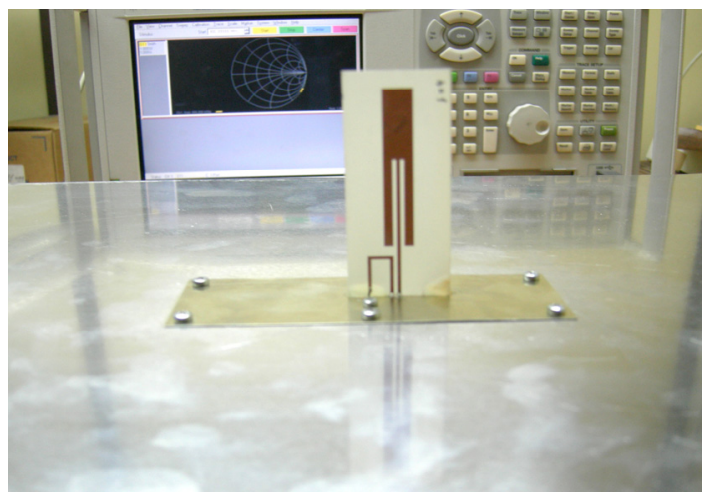

Figure 8. Measuring the UHF RFID antenna input impedance using a network analyzer.

The measured input impedance of the realized UHF RFID antenna at $900 \mathrm{MHz}$ using the "one half method" is $\mathrm{Za} / 2=$ $(10.6+j 76.4) \Omega(\mathrm{Za}=(21.2+\mathrm{j} 152.8) \Omega)$ and is shown in the Smith chart in Fig. 9.

The measured real part of the impedance value deviates from the real part of the ASIC chip input impedance by $1.2 \Omega$; its imaginary part deviates from the corresponding part of the chip impedance by $25.8 \Omega$. This indicates a quite good matching that promises low loss of RF energy.

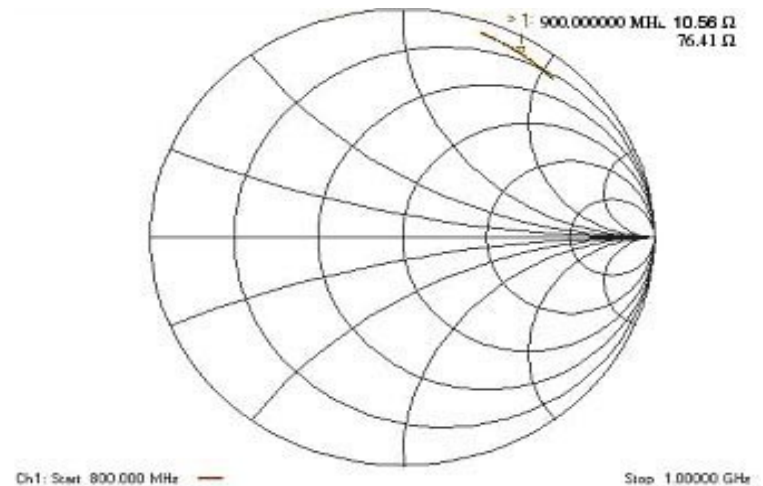

Figure 9. Diagram of the measured half input impedance of the realized UHF RFID antenna in the range 0.8 to $1.0 \mathrm{GHz}$.
Verification of the whole system operation is carried out by measuring the reading distance of the reader (UHFRW-G2-232)-transponder (tag) system. It shows that this distance (measured in an office) is approximately $2 \mathrm{~m}$. The antenna was placed on the cardboard box (object to identify). The overall dimensions of the box were 360 $\mathrm{mm} \times 235 \mathrm{~mm} \times 130 \mathrm{~mm}$.

\section{Conclusion}

The simulated and realized [6] [7] UHF RFID antenna is characterized by a new and simple design as well as the electrical characteristics that completely satisfy standards of the RFID systems. The antenna covers the entire UHF RFID band (Europe, America and Asia). Such simple design implies easy and low-cost manufacturing, especially when realized on inexpensive substrates (PET, PVC) by using screen printing.

\section{References}

[1] Finkenzeller, K."RFID Hand Book", John Wiley \& Sons Ltd. 2003.

[2] Marrocco, G. "The Art of UHF RFID Antenna Design: Impedance-Matching and Size-Reduction Techniques", IEEE Antennas and Propagation Magazine, Vol.50, No1, feb.2008.

[3] John Kraus, "Antennas", McGraw-Hill, Second Edition, 1988, pp.728-729.

[4] Sehagiri Rao, K.V.; Pavel V. Nikitin; Sander F. Lajm, "Antenna Design for UHF RFID Tags: A Review and Protocol Application", IEEE Trans. Antennas Propag., Vol.53, No.12, pp.3870-3876, Dec. 2005.

[5] Nešić, A.; Radnović, I.; Mikavica, M.; Dragaš, S.\& Marjanović, M. "New Printed Antenna with Circular Polarization", EMC-96 Prag, Sept. 1996, Proceedings 1,2, pp.569-570.

[6] Popović, N.; Manojlović, P.; “UHF RFID Antenna: A Printed Dipole Antenna with CPS Matching Circuit and Inductively Coupled Feed", TELSIKS 9 ${ }^{\text {th }}$, International Conference on Telecommunication in Modern Satellite, Cable and Broadcasting Services, Niš, 7-9. Oct. 2009, pp.445-448.

[7] www.wipl-d.com

[8] Griffin, J. D.; Durgin, G.D.; Haldi, A.\& Kippelen, B. "RF Tag Antenna Performance on Various Materials Using Radio Link Budgets", IEEE Antennas and Wireless Prop. Letters, Vol.5, pp.247-250, 2006.

[9] Tikohov, Y.; Kim, Y.\& Min, Y.H."Compact Low Cost Antenna for Passive RFID Transponder", IEEE Antenna and Propagation Society International Symposium 2006, July 2006, pp.1015-1018. 